\title{
Efeito do filtro de ultravioleta em lentes de contato hidrofílicas de alta hidratação
}

\author{
Ultraviolet filter effect on hydrophilic \\ high hydrated contact lenses
}

Arlindo José Freire Portes ${ }^{1}$, César Antonio Elias², Celso Laje ${ }^{3}$, Lívia Faria Jordão ${ }^{4}$, Natasha Moreira Lyrio ${ }^{5}$

\section{ReSUMO}

A exposição à radiação ultravioleta está relacionada ao aparecimento de patologias oculares como ceratites, cataratas, degenerações maculares ou pterígio. Esta radiação está dividida em 3 tipos de acordo com o seu comprimento de onda: Uv-A (320-400nm), Uv-B (290-320nm) e Uv-C (100-290nm). A indústria óptica desenvolveu filtros de ultravioleta para lentes intra-oculares, lentes de contato e lentes de óculos. Objetivo: Avaliar a eficácia do filtro de ultravioleta em lentes de contato de alta hidratação. Métodos: Seis grupos de lentes de contato de alta hidratação e de marcas diferentes, contendo 3 unidades novas e iguais foram estudadas, observando-se o percentual de transmissão da energia luminosa de 200 a $400 \mathrm{~nm}$ através de um espectofotômetro. Resultados: Os 3 grupos que apresentavam filtro de ultravioleta causaram uma queda abrupta na transmissão da radiação ultravioleta de $400 \mathrm{~nm}$ até $340 \mathrm{~nm}$, ocorrendo um aumento na transmissão entre 240 a $300 \mathrm{~nm}$ até um nível máximo de $45 \%$. Abaixo de $240 \mathrm{~nm}$ e entre 300 e $340 \mathrm{~nm}$, houve um completo bloqueio da transmissão da radiação ultravioleta. Os 3 grupos que não apresentavam filtros de ultravioleta causaram uma queda abrupta na transmissão da radiação ultravioleta abaixo de $240 \mathrm{~nm}$, ocorrendo um bloqueio completo de transmissão abaixo de $220 \mathrm{~nm}$. Conclusão: As lentes de contato sem filtro Uv testadas não bloquearam com eficácia a radiação Uv-A, B ou C, e as com filtro bloquearam quase totalmente a radiação Uv-B e parcialmente a radiação Uv-A e Uv-C.

Descritores: Lentes de contato; Raios ultravioleta/efeitos adversos; Filtros; Radiação; Oftalmopatias/etiologia

\footnotetext{
1Doutor, Professor adjunto de Oftalmologia da Universidade Estácio de Sá - Rio de Janeiro (RJ);

${ }_{2}^{2}$ Professor do laboratório de biofísica das refrações do Instituto Carlos Chagas na UFRJ - Rio de Janeiro (RJ);

${ }^{3}$ Professor de Biofísica do Instituto Carlos Chagas da UFRJ - Rio de Janeiro (RJ);

${ }^{4}$ Acadêmica do $6^{\circ}$ ano de medicina da Universidade Estácio de Sá - Rio de Janeiro (RJ);

${ }^{5}$ Acadêmica do $6^{\circ}$ ano de medicina da Universidade Estácio de Sá - Rio de Janeiro (RJ).
}

Trabalho realizado no Instituto Carlos Chagas da UFRJ 


\section{INTRODUÇÃO}

D esde a introdução de lentes de contato descartáveis em 1987, as vantagens destas têm sido publicadas, e novos benefícios a saúde ocular foram introduzidos, como é o caso dos filtros de ultravioleta ${ }^{(1)}$.

Existem na literatura evidências que associam a radiação ultravioleta com patologias que afetam os olhos. Os sinais e sintomas mais comuns, determinados por uma exposição aguda, são hiperemia, lacrimejamento intenso, prurido, fotofobia, edema conjuntival e palpebral e dificuldade de adaptação ao escuro ${ }^{(2)}$. Sabe-se há muito tempo que a exposição à radiação ultravioleta causa uma modificação no epitélio da conjuntiva, levando ao desenvolvimento de pterígio. A radiação ultravioleta também pode induzir a uma fotoceratite, vários estudos falam sobre perdas das camadas celulares do epitélio, envolvimento do estroma e de células endoteliais, o que pode, além de uma ceratite, comprometer futuras cirurgias intra-oculares, pelo comprometimento endotelial $^{(3-7)}$.

O desenvolvimento da catarata devido à exposição da radiação ultravioleta foi demonstrado em animais de laboratórios e a associação de catarata com a exposição à altas doses de radiação ultravioleta também está estabelecida ${ }^{(3-7)}$.

Embora pouca quantidade de radiação ultravioleta atinja a retina, há estudos que a correlacionam com o desenvolvimento de degeneração macular relacionada a ida$\mathrm{de}^{(2,8-9)}$. Recentemente, lentes de contato hidrofílicas com filtro ultravioleta foram introduzidas no mercado como uma opção para a proteção ocular. Estas lentes, quando adequa- damente adaptadas, cobrem toda a córnea, as paliçadas de Vogt e parcialmente a conjuntiva, protegendo, conseqüentemente, a córnea, o cristalino e a retina. A lente também protege o limbo, que é o local onde se encontram as células germinativas, sendo estas fonte de novas células epiteliais para a córnea. Quando comparadas com as lentes rígidas, as lentes gelatinosas conferem maior proteção aos efeitos deletérios do ultravioleta devido ao seu maior diâmetro, recobrindo um maior número de estruturas oculares ${ }^{(10)}$.

O nosso trabalho tem como objetivo avaliar o efeito do filtro de ultravioleta em lentes de contato hidrofílicas de alta hidratação.

\section{Métodos}

Foram utilizados seis grupos de lentes de contato hidrofílicas de alta hidratação, cada qual com 3 unidades novas e iguais, e os grupos apresentavam lentes de contato com as características expressa na Tabela 1.

Foi usado um espectrofotômetro da marca Milton Hoy, modelo Genesis II, constituído de uma unidade monocromadora que emite radiação ultravioleta variando de 200 a 400 nanômetros (nm) na direção de uma célula fotoelétrica, que capta o percentual de energia transmitida através de determinado material situado entre ela e a unidade monocromadora.

Cubetas de quartzo de $3 \mathrm{ml}$ foram usadas para colocar o soro fisiológico e a lente de contato a ser analisada.

Uma cubeta de quartzo (material que permite a transmissão de cerca de $100 \%$ da radiação ultravioleta) foi preenchida com soro fisiológico e colocada no espectrofotômetro entre a unidade monocromadora e a

Tabela 1

Lentes de contato hidrofílicas de alta hidratação

\begin{tabular}{|c|c|c|c|c|c|c|}
\hline Grupos & Material & $\begin{array}{c}\text { Curva Base } \\
(\mathbf{m m})\end{array}$ & $\begin{array}{c}\text { Diâmetro } \\
\text { (mm) }\end{array}$ & $\begin{array}{c}\text { Conteúdo de } \\
\text { água }\end{array}$ & $\begin{array}{c}\text { Grau } \\
\text { D* }\end{array}$ & $\begin{array}{c}\text { Filtro } \\
\text { ultravioleta }\end{array}$ \\
\hline Grupo 1 & Etalficon A & 8.8 & 14.00 & $58 \%$ & -3.00 & Ausente \\
\hline Grupo 2 & Oculficom D & 8.6 & 14.20 & $55 \%$ & -3.00 & Ausente \\
\hline Grupo 3 & $\begin{array}{l}\text { Copolímero de } \\
\text { hidroxietilmetacrilato } \\
\text { e metacrilato de glicerol }\end{array}$ & 8.7 & 14.00 & $55 \%$ & -3.00 & Ausente \\
\hline Grupo 4 & Etalficon A & 8.8 & 14.00 & $58 \%$ & -3.00 & Presente \\
\hline Grupo 5 & $\begin{array}{c}\text { Polímero de } \\
\text { metilmetacrilato } \\
\text { N-vinil-2-pirolidona }\end{array}$ & 8.7 & 14.40 & $74 \%$ & -3.00 & Presente \\
\hline Grupo 6 & Vasurfilcon A & 8.7 & 14.40 & $74 \%$ & -3.00 & Presente \\
\hline
\end{tabular}

Legenda: *D - Dioptrias 
célula fotoelétrica. Energia radiante de comprimento de onda variando de 200 a $400 \mathrm{~nm}$ foi transmitida através da cubeta e o percentual de transmissão de cada comprimento de onda foi determinado e considerado padrão, equivalente a $100 \%$ para as condições experimentais testadas.

Uma lente de contato do grupo 1 foi colocada dentro da cubeta de quartzo, preenchida com soro fisiológico e colocada entre a unidade monocromadora e a célula fotoelétrica. Energia radiante ultravioleta variando de 200 a $400 \mathrm{~nm}$ foi transmitida através da lente na cubeta e o percentual de transmissão de cada comprimento de onda foi determinado pelo aparelho em relação ao padrão fixado na medição anterior como mostra a Figura1.

$\mathrm{O}$ mesmo processo se repetiu com outras lentes de contato do experimento e para cada lente foi impresso um gráfico de percentual de transmissão da radiação ultravioleta.

\section{Resultados}

Os gráficos, avaliando o percentual de transmissão de energia radiante em função dos comprimentos de onda, nas lentes de contato de cada um dos grupos testados apresentaram-se semelhantes, indicando um padrão industrial característico de cada marca para a transmissão da radiação ultravioleta.

Observou-se no nosso estudo que as lentes sem filtro de ultravioleta causaram uma queda abrupta na transmissão da radiação ultravioleta em aproximadamente $240 \mathrm{~nm}$, ocorrendo um bloqueio completo de transmissão abaixo de 220nm. A Figura 2 apresenta os percentuais de transmissão da radiação ultravioleta em função do comprimento de onda para as lentes de contato dos grupos 1, 2 e 3 respectivamente.

Os valores característicos da transmissão da radiação ultravioleta para as lentes de contato com filtro do grupo 4, 5 e 6 estão representados abaixo na Figura 3.

As lentes com filtro ultravioleta causaram uma queda abrupta na transmissão da radiação ultravioleta de $400 \mathrm{~nm}$ até $340 \mathrm{~nm}$, ocorrendo um aumento na transmissão entre 240 a 300 nm até um nível máximo de $45 \%$.

Abaixo de $240 \mathrm{~nm}$ e entre $300 \mathrm{~nm}$ e $340 \mathrm{~nm}$, houve um completo bloqueio da transmissão da radiação ultravioleta.

\section{Discussão}

As lentes de contato com filtro de ultravioleta são produzidas para protegerem as estruturas oculares do Uv-A, Uv-B e Uv-C.A maior parte da radiação Uv-A é

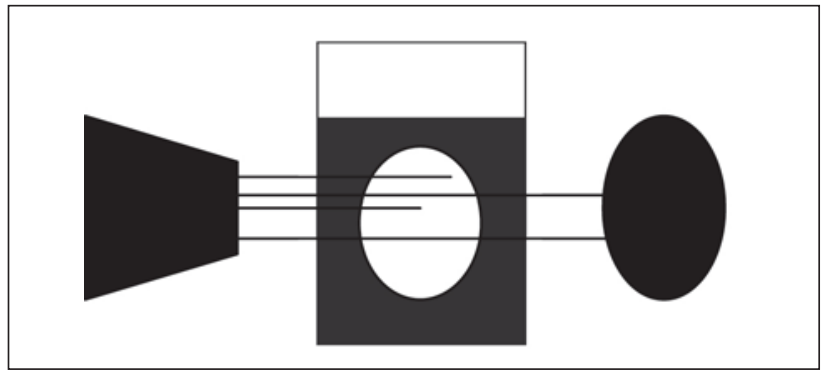

Figura 1: Lente de contato do grupo 1 colocada numa cubeta de quartzo com soro fisiológico entre a unidade monocromadora e a célula fotoelétrica

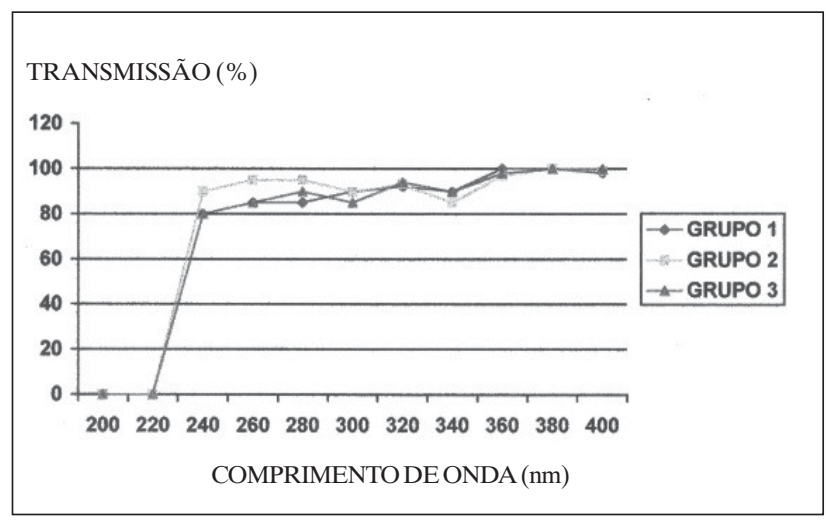

Figura 2: Percentual de transmissão da radiação ultravioleta em função do comprimento de onda das lentes de contato dos grupos 1 , 2 e 3

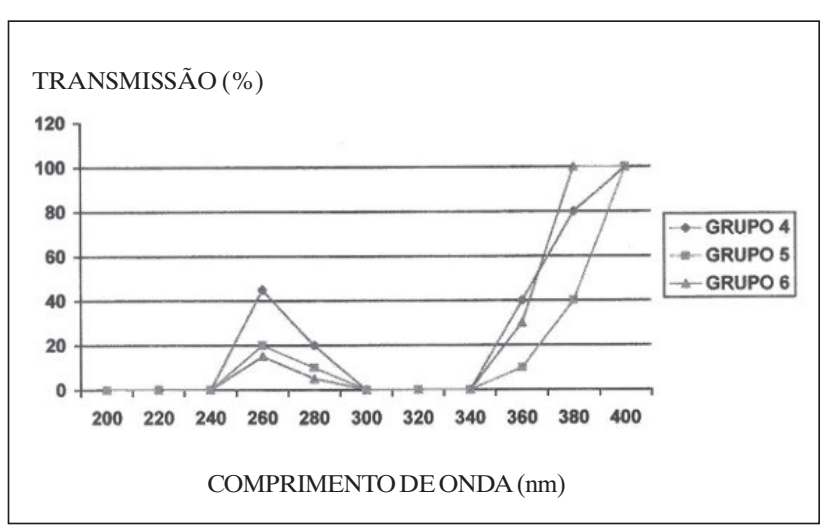

Figura 3: Percentual de transmissão da radiação ultravioleta em função do comprimento de onda das lentes de contato dos grupos 4 , 5 e 6

absorvida no cristalino, conseqüentemente, a retina é protegida, entretanto em pacientes afácicos ou com lentes intra-oculares antigas esta radiação é transmitida à retina.

A radiação Uv-B, por outro lado,é absorvida primariamente pela córnea e conjuntiva, com $10 \%$ de penetração na superfície anterior do cristalino. Esta absorção por tecidos oculares pode causar ceratoconjuntivite, pinguéculas,pterígio, cataratas corticais e subcapsulares. Já a radiação Uv-C é 
absorvida em condições normais na atmosfera.

Vários trabalhos têm sido feitos para analisar se as lentes de contato hidrofílicas com filtro ultravioleta protegem as várias estruturas oculares e quais são os comprimentos de onda do espectro eletromagnético absorvidos por esses filtros ${ }^{(3-5,7-8,8,11)}$.

Uma análise de lentes de contato classificadas pelos fabricantes, como com filtro ultravioleta mostrou que as lentes de material Etafilcon A com filtro, bloqueiam parte do espectro da radiação ultravioleta exibindo transmissão na faixa do Uv-C, atingindo um pico entre $260-267 \mathrm{~nm}{ }^{(10)}$. Porém, nas lentes de Etafilcon A sem filtro, a transmissão teve início em $235 \mathrm{~nm}$, não conferindo nenhuma proteção na faixa do Uv-A e Uv-B. Resultados semelhantes foram obtidos em nosso estudo.

Em estudo comparativo clínico entre lentes de Etalficon A com filtro de ultravioleta e as sem filtro de ultravioleta, as lentes de contato com filtro bloquearam $97.5 \%$ do Uv-B e $83.4 \%$ do Uv-A, havendo bloqueio da transmissão de radiação ultravioleta de 380 $\mathrm{nm}$ a $200 \mathrm{~nm}$, sendo que entre $225 \mathrm{~nm}$ e $280 \mathrm{~nm}$ havia transmissão parcial de até $35 \%$ da radiação ultravioleta

${ }^{(11)}$. Clinicamente não houve alterações entre pacientes que usaram os dois tipos de lentes. No nosso estudo também foi avaliado o percentual de transmissão da radiação ultravioleta em lentes de Etalficon A sem filtro e com filtro, os resultados foram semelhantes, exceto na faixa de transmissão do Uv-A, visto que no nosso estudo o percentual de transmissão nas lentes com filtro foi significativamente maior que o observado no trabalho de Hickson-Curran.

Rabello et al. estudou a transmissão da radiação ultravioleta em lentes de óculos solares com filtro e sem filtro UV e observou que as lentes de óculos que possuíam filtro ultravioleta eram capazes de bloquear a transmissão da incidência frontal da radiação, porém não impediam a passagem da incidência oblíqua e periférica da radiação entre a lente e a haste da armação ${ }^{12}$. A proteção para esta forma de incidência da radiação é conferida pelas lentes de contato gelatinosas com filtro UV, devido ao seu contato direto com a estrutura ocular. Portanto, é recomendável que as pessoas que fazem uso de óculos e se expõem à radiação UV, dêem preferência aos óculos com hastes mais grossas. As lentes de contato por sua vez, não protegem os olhos da incidência de radiação Uv na conjuntiva ou pálpebras, não substituindo conseqüentemente o uso de óculos de sol com filtro.

Além do sol, o homem é constantemente exposto a uma variedade de fontes de radiação artificial que emitam, além de luz visível, radiação de ultravioleta, por exemplo: lâmpadas incandescentes, lâmpadas de mercúrio, lâmpadas fluorescentes, lâmpadas germicidas.

No nosso estudo, observamos que a radiação Uv$\mathrm{B}$ foi eficientemente bloqueada pelos filtros testados. A radiação Uv-C foi bloqueada parcialmente pelos filtros testados, porém ela não atinge normalmente a superfície da terra sendo filtrada quase que totalmente pela camada de ozônio da estratosfera. Portanto, aquelas pessoas que estão em localizações geográficas onde a camada de ozônio está diminuída ou que trabalhem com lâmpadas germicidas ou ainda de mercúrio, serão expostas a radiação ultravioleta mesmo que usem as lentes testadas com filtros. Portanto, indivíduos que se exponham ao Uv-C podem necessitar de proteção adicional para os olhos ${ }^{(7)}$.

Nas caixas comerciais de lentes de contato com filtro de ultravioleta, poderiam haver especificações referentes a eventual transmissão da radiação Uv-C, para alertar os usuários de fontes artificiais desta radiação e moradores de áreas geográficas expostas a abertura na camada de ozônio da atmosfera.

Os filtros de ultravioleta podem evoluir em um futuro próximo para a absorção total desta radiação, tornando a incidência de doenças causadas por exposição ao ultravioleta extintas.

\section{Conclusão}

1 - As lentes de contato de alta hidratação, com filtro de ultravioleta testadas, bloquearam de forma incompleta a radiação ultravioleta $\mathrm{A}$ (comprimento de onda entre $400 \mathrm{~nm}$ a $320 \mathrm{~nm}$ ) e C (comprimento de onda de $280 \mathrm{~nm}$ a $100 \mathrm{~nm}$ ), e com eficácia a radiação ultravioleta B (comprimento de onda entre $320 \mathrm{~nm}$ e $280 \mathrm{~nm}$ ).

2 - As lentes de contato de alta hidratação sem filtro de ultravioleta bloquearam de forma completa apenas a radiação ultravioleta no comprimento de onda de $200 \mathrm{~nm}$ a $220 \mathrm{~nm}$, transmitindo intensidades significativas entre $240 \mathrm{~nm}$ e $400 \mathrm{~nm}$ nas faixas do UvA, Uv-B e Uv-C.

\section{Abstract}

The exposure to ultraviolet radiation is associated with the beginning of ocular pathologies like keratites, cataract, age-related macular degeneration and pterygium. This radiation is divided in 3 bands according to the wave length: Uv-A (320-400), Uv-B (290-320), Uv-C (100-290). The optic industry developed ultraviolet filters to intraocular lenses, contact lenses and spectacle lenses. 
Purpose: The issue of this work is to estimate the efficacy of ultraviolet contact lenses with high hydration. Methods: Six types of high hydrated contact lenses from different brands with three new equal units were evaluated, observing the transmittance rates of light energy in the wave bands between 200 to $400 \mathrm{~nm}$ measured with spectrophotometer. Results: Three groups that had ultraviolet filters blocked sharply the Uv transmittance from $400 \mathrm{~nm}$ to $340 \mathrm{~nm}$. There was an increase in $U v$ transmittance between $240 \mathrm{~nm}$ to $300 \mathrm{~nm}$ up to $45 \%$. Below $240 \mathrm{~nm}$ and between $300 \mathrm{~nm}$ and $320 \mathrm{~nm}$ there was a complete Uv block. The three contact lenses types that didn't have ultraviolet filters block sharply Uv radiation below $240 \mathrm{~nm}$ and below $220 \mathrm{~nm}$ there was a complete $U v$ block. Conclusion: Contact lenses that diddn't have UV filter didin't block efficaciously $U v-A, U v-B$ or $U v-C$. The ultraviolet filter have blocked the $U v-B$ radiation almost completely, and partially the $U v-A$ and $U v-c$.

Keywords: Contact lenses ; Ultraviolet rays/adverse effects; Filters; Radiation; Eye diseases/etiology

\section{ReFERÊNCIAS}

1. Hamano H, Watanabe K, Hamano T, Mitsunuga S, Kotani S, Okada A. A study of the complications induced by conventional and disposable contact lenses. CLAO J. 1994; 20(2):103-8. Comment in: CLAO J. 1994; 20(2):86-7.

2. Oliveira PR, Oliveira AC, Oliveira FC. A radiação ultravioleta e as lentes fotocrômicas. Arq Bras Oftalmol. 2001; 64(2):163-5.

3. Bergmanson JP, Söderberg PG. The significance of ultraviolet radiation for eye diseases. A review with comments on the efficacy of UV-blocking contact lenses. Ophthalmic Physiol Opt. 1995;15(2): 83-91.
4. Bergmanson JP, Pitts DG, Chu LW. Protection from harmful UV radiation by contact lenses. J Am Optom Assoc. 1988; 59(3): 178-82.

5. Pitts DG, Lattimore MR. Protection against UVR using the Vistakon UV-Bloc soft contact lens. Int Contact Lens Clin. 1987; 14(1): 22-9.

6. Pitts DG, Bergmanson JP, CHU LW. Ultrastructural analysis of corneal exposure to UV radiation. Arch Ophthalmol (Copenh). 1987; 65(3): 263-73.

7. Bergmanson JP, Sheldon TM. Ultraviolet radiation revisited. CLAO J. 1997; 23(3): 196-204.

8. Cruickshanks KJ, Klein R, Klein BE. Sunlight and age-related macular degeneration. The Beaver Dam Eye Study. Arch Ophthalmol. 1993; 111(4): 514-8.

9. de Jong PT. Age-related macular degeneration. N Engl J Med. 2006; 355(14):1474-85. Comment in: N Engl J Med. 2006; 355(14):1493-5.

10. Giasson CJ, Quesnel NM, Boisjoly H. The ABCs of ultravioletblocking contact lenses: an ocular panacea for ozone loss? Int Ophthamol Clin. 2005; 45(1):117-39.

11. Hickson-Curran SB, Nason RJ, Becherer PD, Davis RA, Pfeifer J, Stiegemeier MJ. Clinical evaluation of Acuvue contact lenses with UV blocking characteristics. Optom Vis Sci. 1997; 74(8): 632-8.

12. Rabello MS. Os principais danos ocorridos em tecidos oculares relacionados à radiação ultravioleta e os métodos de proteção. Novo Enfoque. 2007 [citado 2008 Jan 12]; 5(5):[cerca de 15p.] .Disponível em:http://www.castelobranco.br/pesquisa/vol5/PDFs/07.pdf
ENDREÇO PARA CORRESPONDÊNCIA
Arlindo José Freire Portes
Av. Nossa Senhora de Copacabana, 195 / 409
CEP 22020-002 - Rio de Janeiro - RJ
Tel: (21) 22544398 / 2541-4532
e-mail : portes@uol.com.br 